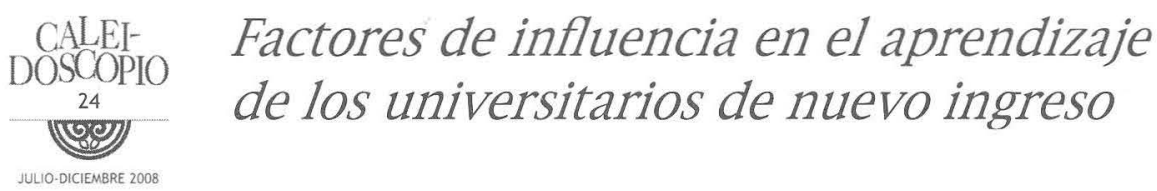

DRA. GABRIELA HERNÁNDEZ ZAPATA' / MTRA. BEATRIZ ALEJANDRA GONZÁLFZ MEDINA

/MTRO. JOSÉ ANTONIO VÁZQUEZ IBARRA/MTRO. MIGUEL ÁNGEL ROMO MARTÍNEZ

/MTRO. RODOLFO RAFAEL MEDINA RAMÍREZ

Universidad Politécnica de Aguascalientes

INTRODUCCIÓN

El propósito de este trabajo fue identificar algunos de los factores que especialmente intervienen en el desempeño de los estudiantes universitarios: expectativas educativas y ocupacionales, hábitos de estudio, orientación profesional, aspectos socioeconómicos, valoración familiar del estudio, y desempeño docente. Con base en los resultados obtenidos se orientarán acciones para guiar a los alumnos en el mejoramiento de sus hábitos de estudio a través de programas de formación o asesoramiento continuo, de acuerdo a las necesidades específicas, cuyo efecto tenga repercusión efectiva a lo largo de su trayectoria universitaria. A tal efecto se aplicó una encuesta de 53 preguntas y un cuestionario de 70 preguntas ${ }^{2}$ a 295 alumnos de nuevo ingreso a la Universidad Politécnica de Aguascalientes (UPA), en el ciclo septiembre - diciembre de 2007.

Departamento de Pedagogía. Universidad Politécnica de Aguascalientes. Prolongación Mahatma Gandhi Km. 2. Tel. (449) 442 1400, e-mail: gabriela.hernandez@ upa.edu.mx

2 De Garay Sánchez: 2001

C A L E

D O S C

C O P ।

○ 
El procedimiento para el análisis de los datos mencionados fue la agrupación de los factores de influencia en el desempeño académico. La aplicación vía electrónica del cuestionario y la encuesta se llevó a cabo durante las dos primeras semanas del cuatrimestre, con la escala de respuestas que se muestra a continuación: siempre, casi siempre, a veces, casi nunca, nunca.

Para el estudio sólo se tomaron en cuenta las respuestas con los porcentajes más altos, o los más bajos, pues en este rango se ubicaron los casos de estudio más útiles o significativos.

\section{RESULTADOS Y DISCUSIÓN}

Es conocido el hecho en el desfase existente entre los conocimientos, habilidades, métodos de estudio, desempeño académico entre el nivel medio superior y superior. En consecuencia, se agrega a la lista de las funciones de la Universidad, desarrollar estrategias para nivelar académicamente a los estudiantes de nuevo ingreso y prevenir la deserción escolar, fenómeno que suele presentarse con mayor frecuencia en el primer año del nivel superior, así lo sustentan varios autores: Tejedor, Javier; González Alfonso, Miriam; Álvarez, Pedro y otros³. En este sentido, se ha pretendido detectar, en primer término, cuáles son los factores que intervienen en el desempeño de los estudiantes y el nivel de influencia en los resultados de sus estudios a fin de identificar las estrategias a reforzar o implementar en la UPA, con la intención de generalizarlas, según la pertinencia y necesidades específicas de cada institución de educación superior.

3 Javier Tejedor, "Poder explicativo de algunos determinantes del rendimiento en los estudios universitarios", en Revista española de Pedagogía, vol. LXI, No. 224, (Enero-abril 2003); Catalina González Alfonso et. al., "El abandono de los estudios universitarios: factores determinantes y medidas preventivas", en Revista española de Pedagogía, vol. LXV, No. 236, 071-086, Enero-abril, 2007. 


\subsection{Factores que influyen en el rendimiento académico}

De acuerdo a la experiencia docente, la motivación que más puede determinar el rendimiento académico es la intrínseca, debido a que en condiciones normales, la voluntad, el querer decidir, mueve a la ejecución. El apoyo moral, el acompañamiento, de otra persona o grupos de personas contribuye en el esfuerzo, pero no en el nivel de querer actuar por libre albedrío o vocación. En este sentido, las motivaciones tienen correlación con expectativas satisfechas, así por ejemplo: hay universitarios con confianza en sí mismos para encontrar un empleo al terminar sus estudios, además conocen las implicaciones de esfuerzo permanente para conseguirlo: asistir a clases, elaborar proyectos, atender a los profesores, entregar puntualmente las evidencias de aprendizaje, entre otros.

\section{EXPECTATIVAS DE LOS ESTUDIANTES AL INICIAR LA CARRERA Y OPORTUNIDADES DE EMPLEO FUTURO}

Las inferencias se corroboran al observar que los estudiantes consideran muy satisfechas las expectativas hechas al iniciar su carrera 40.28\%, satisfactorias $51.24 \%$ y un mínimo, $6.36 \%$ poco satisfactorias. En cuanto a las oportunidades de empleo futuro, reflejan una proyección positiva sobre la confianza de lograr una formación de calidad que les permita lograr empleo al terminar sus estudios. La autoestima saludable también se relaciona con el establecimiento de metas de aprendizaje $e^{4}$ o con un propósito.

\section{HÁBITOS Y PRÁCTICAS ESCOLARES}

En orden de frecuencia descendente, se describen a continuación los hábitos y prácticas escolares que siempre realizan los estudiantes que ingresaron en septiembre de 2007.

4 Javier Tejedor, op. cit.

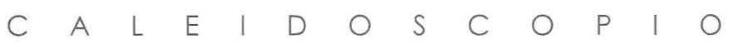


- Asistir a clases (84.12\%). Acto que se explica porque en la UPA es obligatorio el pase de lista.

- Asistir puntualmente (67.61\%). Este hábito se encuentra más o menos arraigado porque por reglamento a la hora en punto se debe comenzar el pase de lista. Sólo se tiene derecho a faltar el 10\% de las horas totales por materia.

- Escuchar a los maestros (55.41\%). Los demás pueden tener dificultades de concentración

- Tomar apuntes (47.97\%). Una estrategia para concentrarse es tomar apuntes y es una práctica de menos de la mitad de los estudiantes.

- Tomar dictado (42.91\%). Parece que es casi igual de recurrente tomar dictado y tomar apuntes, por tanto, es posible la falta de reflexión, de escucha activa y, en consecuencia, de comprensión.

- Realizar preguntas en clases (17.91\%). Si falta escuchar activamente y comprender, es escasa la materia disponible a cuestionar.

- Discutir los puntos de vista del profesor (14.84\%). La causa puede relacionarse con los indicadores anteriores y se prescinde de realizar lectura previa.

- Preparar la clase $(8.11 \%)$. Es escaso el grupo de estudiantes que preparan o repasan la clase antes de iniciar.

- Discutir con base en una lectura previa (6.76\%). La razón más probable puede ser la falta de lectura previa. Se confirma la carencia del hábito de la lectura.

- Discutir sin base en una lectura previa. (4.05\%). Es evidente la falta de la práctica de la discusión en clase, independientemente de la estrategia utilizada. 
Gráfica 1. HÁBITOS Y PRÁCTICAS ESCOLARES QUE SIEMPRE REALIZAN LOS ESTUDIANTES. ${ }^{5}$

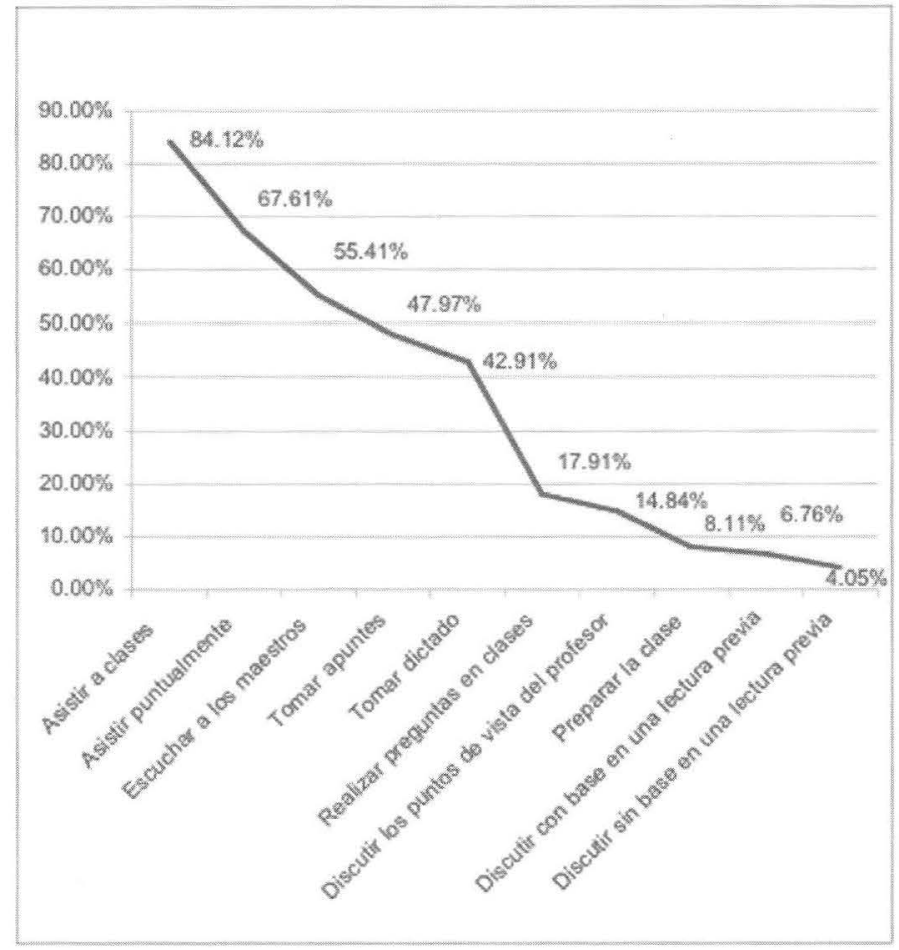

Los hábitos y prácticas escolares pueden predecir el rendimiento en los estudios puesto que la disciplina, perseverancia, orden, responsabilidad, serán fundamentales y continuamente necesarios en el éxito, en cualquier contexto personal, familiar, social y escolar. Lógicamente se deducen las consecuencias de la ausencia de los hábitos positivos, propiciando en ocasiones, tomar decisiones extremas, como el abandono o la deserción escolar.

5 Fuente: Encuesta aplicada a 295 alumnos de nuevo ingreso a la Universidad Politécnica de Aguascalientes (UPA), en el ciclo septiembre - diciembre de 2007. Los alumnos podían seleccionar más de una respuesta. 


\section{DÓNDE OBTIENEN LOS MATERIALES PARA REALIZAR SUS LECTURAS}

El uso intensivo del Internet como fuente de información para la elaboración de trabajos académicos (46.28\%), muestra el fuerte impacto que ha tenido el uso de los medios tecnológicos en los universitarios actuales. La restricción en esta fuente de información será que el alumno tenga la capacidad para distinguir entre fuentes confiables y fuentes no confiables. Con estos resultados se evidencia la falta de práctica de acudir a la biblioteca de la universidad (36.15\%) y sólo el $11.82 \%$ acude a otras bibliotecas. Es escasa también la práctica de la compra y prestamos de libros (6.42\% y $11.49 \%$, respectivamente). Es más frecuente recurrir a las fotocopias de los textos (31.08\%). Ver gráfica 2.

GRÁFICA 2. DÓNDE OBTIENEN LOS MATERIALES PARA REALIZAR SUS LECTURAS. $^{6}$

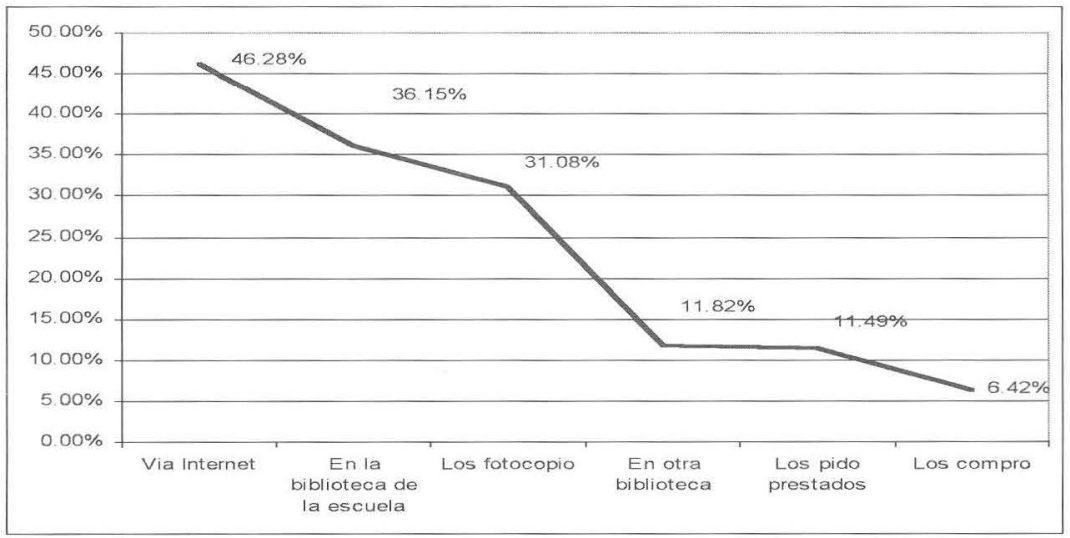

6 Fuente: Encuesta aplicada a 295 alumnos de nuevo ingreso a la Universidad Politécnica de Aguascalientes (UPA), en el ciclo septiembre - diciembre de 2007. 
Es posible que la poca compra de libros se deba a la utilización de los medios electrónicos para realizar consultas. Incluso es probable que el tipo de material impreso existente en los hogares no tenga relación con los tópicos de las materias de estudio formal.

\section{ESTRATEGIAS QUE UTILIZAN PARA REALIZAR SUS LECTURAS}

Locke sostiene que la evidencia de un inadecuado desempeño en el rendimiento académico no se debe únicamente a la falta de organización del tiempo.

"Los hechos indican que los alumnos que no tienen éxito...emplean métodos de estudio ineficientes, de modo que entienden muy poco de lo que estudian y recuerdan muy poco de lo que entienden"7.

Asimismo, el restar importancia a los hábitos de estudio provoca las dificultades académicas, especialmente en el primer año de la formación universitaria, periodo en el que suele presentarse el mayor índice de deserción. En este sentido, es patente la necesidad de emplear estrategias o métodos de estudio encaminados a lograr un mejor desempeño puesto que las estrategias menos utilizadas son las que involucran mayor esfuerzo intelectual e inversión de tiempo: esquemas (35\%); notas al margen (27\%); cuestionarios (22.6\%); diagramas (22.3\%); fichas (15.88\%).

Las estrategias intelectuales más utilizadas: subrayado $(80.74 \%)$; resúmenes (69.26\%) son recomendables, siempre y cuando se utilicen como apoyos a las estrategias más complejas para el logro del aprendizaje significativo. Además, los métodos y estrategias específicas de estudio pueden guiarse en el proceso de enseñanza, a través del uso

7 Edwin Locke, Guia para estudiar. México, Diana, 1987.

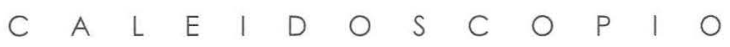


de la tecnología como herramienta didáctica ${ }^{8}$ y a su vez, como medio de aprendizaje, de modo que se aprovechen los recursos y estrategias nuevas y tradicionales efectivas sin perder de vista el objetivo del aprendizaje significativo.

\section{DESEMPEÑO DOCENTE}

La importancia de la docencia en la universidad es reconocida por todos los actores involucrados en el sector de la educación formal ${ }^{9}$. La trascendencia de la labor de los profesores radica en que guían el proceso de aprendizaje de los estudiantes, con la finalidad de formarlos para la vida profesional, laboral y social ${ }^{10}$. Por tanto, la calidad del desempeño docente implica la capacitación profesional pertinente, vocación de servicio, dominio de los temas, habilidades de comunicación, habilidades de uso de las tecnologías de la información y la comunicación, etc.

Sin embargo, la capacidad de un profesor se deriva esencialmente del aprendizaje de sus alumnos. El aprendizaje se facilita a los jóvenes a través de diversos medios didácticos, organización de actividades, evaluación objetiva, planeación de las sesiones, etc. Es interesante la percepción de los alumnos de nuevo ingreso respecto al desempeño de sus profesores, precisamente en la metodología que utilizan para operar el proceso de enseñanza - aprendizaje. Ellos opinan respecto al procedimiento general de organización de la actividad docente que

8 Marcela Santillán Nieto, "Tecnologías de la información y de la comunicación en la educación", en Red Revista Mexicana de Investigación Educativa, 2006; Julio Cabero Almenara, Formación del profesorado universitario en estrategias metodológicas para la incorporación del aprendizaje en red en el espacio de educación superior (EEES), Sevilla, Universidad de Sevilla, 2007.

9 Cristina Benítez, Reseña de 'Desafíos de la universidad contemporánea. Los casos de Alemania, estados unidos y América Latina" de Renate Marsiske, en Red Investigación Educativa, 2006, p. 4. http://site.ebrary.com/lib/univeraguascalientessp/

10 Mario Rueda Beltrán, Evaluación de la labor docente en el aula universitaria, México, Universidad Nacional Autónoma de México, 2006. 
los maestros siempre exponen (38.51\%); a veces los alumnos exponen (61.82\%); a veces los maestros dictan (46.62\%); a veces intervienen distintos alumnos (31.08\%). Es palpable la insuficiente incitación a ceder el protagonismo a los estudiantes en la construcción de su propio conocimiento y desarrollo de sus habilidades intelectuales para el aprendizaje significativo.

Además los jóvenes perciben que en sus estudios superiores se fomenta siempre la creatividad (47\%), y la cooperación entre los estudiantes (44.26\%). En general, observan que la calificación final de sus materias es producto de evaluaciones periódicas (89.86\%). En consecuencia, los profesores deberán centrar su atención en lograr los resultados de aprendizaje esperados propiciando que los universitarios se hagan cargo de las responsabilidades que les competen: desarrollo de su creatividad, cooperación, participación, etc.

\subsection{FACTORES OUE INTERVIENEN EN LA DECISIÓN DE CURSAR LA CARRERA, CONSIDERADOS MUY IMPORTANTES}

Influencia de la valoración familiar

Una de las influencias en los estudios de los universitarios es la valoración familiar, específicamente los padres juegan distintos papeles, según el caso particular, los roles pueden ser de: apoyo, indiferencia, o incluso desaprobación, aunque parece ser que la mayor parte de los progenitores pueden sentirse orgullosos de saber que su hijo o hijos estudian una carrera universitaria. El hecho de la relación entre la valoración familiar y los estudios, se explica porque los primeros contactos biológicos y afectivos se originan en la familia, y la seguridad adquirida para tomar decisiones depende en gran parte de la dinámica familiar, fundamentalmente en la infancia.

En este sentido, los resultados de la encuesta confirman el engarce específico de las conversaciones familiares en el proceso de tomar la decisión para cursar la carrera (44.17\%), aunque no tan patente como el gusto personal (71.73\%) y la vocación (46.64\%), debido a la lógica autonomía lograda en los jóvenes por la formación de las propias convicciones y criterios de comportamiento. La influencia de los 
padres es mayor que la información del programa (39.58\%), aunque notoriamente menor que el gusto personal y ligeramente menor que por vocación.

Otras respuestas obtenidas de la investigación de campo que corroboran la relación entre el apoyo familiar y la toma de decisiones en el estudio responden a los cuestionamientos:

1. Qué lugar que ocupa dentro de las prioridades de la familia tus estudios: $65.37 \%$ muy alto, $26.50 \%$ alto.

2. En comparación con tu padre o la persona que ocupa el lugar de jefe de familia cómo percibes el desarrollo de tu vida profesional una vez que concluyas tus estudios: $80.57 \%$ mejor.

3. Económicamente depende alguien de ti: $22.61 \%$ si. El porcentaje de estudiantes que sostienen económicamente a algún miembro o miembros de la familia de origen o propia es significativo.

4. No cuenta en su casa con un espacio privado para estudiar $57.95 \%$. Se infiere que el factor económico limita para disponer en casa de un lugar especial para el estudio, no tanto por la falta de interés de los padres en que los hijos cuenten con las condiciones físicas necesarias para ello. Lo anterior se puede corroborar con que sólo el $8.83 \%$ de los alumnos cuentan con recursos económicos suficientes para estudiar, $76.33 \%$ manifiestan que son suficientes y $13.07 \%$ insuficientes.

La situación socioeconómica puede ser una causa de bajo desempeño académico. Cuando una o varias personas dependen económicamente de un estudiante, es posible que la presión por solventar el gasto familiar se convierta en un factor determinante para abandonar los estudios. De los estudiantes que respondieron la encuesta, $22.61 \%$ son responsables del sustento económico de una o más personas, el porcentaje es considerable y convendría identificar sí está al alcance de la Universidad ofrecer quizá un esquema para trabajadores, ya que el $28.62 \%$ de los estudiantes manifiestan trabajar, lo cual puede explicar la proporción de alumnos que tienen el compromiso con los familiares que dependen económicamente de ellos. Las becas y créditos educativos son medidas que deber reforzarse en las universidades para prevenir la deserción a razón del factor económico.

Por último, los alumnos manifestaron que ha sido mucho el grado en el que ha cambiado su vida al ingresar a la educación superior, en 
los siguientes rubros:

- Exigencia académica $73.85 \%$

- Ambiente social y cultural $51.59 \%$

- Relación familiar $40.28 \%$

- Relación con amistades $46.64 \%$

- Relación con maestros 38.52\%

Las cifras indican la existencia de una patente necesidad de desarrollar programas específicos que contribuyan al fortalecimiento de los hábitos de estudio a fin de lograr un desempeño académico de calidad para favorecer las habilidades, conocimientos y actitudes durante la trayectoria universitaria'!, las cuales se verán reflejadas en el ámbito profesional.

Es común que aproximadamente el $50 \%$ de los estudiantes universitarios terminan satisfactoriamente sus estudios, esto se debe en gran parte a la necesidad de poseer habilidades para aprender a aprender.

\section{CONCLUSIÓN}

Los hábitos se forman desde un arraigo familiar, es por ello que en ocasiones el orden, la perseverancia, el respeto, la comprensión se practique más en unas personas que en otras. Por tanto, los actos operativos que permanecen o llegan a formar parte de la manera de ser de alguien encuentran su origen no en el nivel educativo formal inmediato, sino desde mucho antes, en la infancia.

¿Qué corresponde hacer en la universidad?

Es necesario reforzar el fomento de la utilización de la biblioteca virtual y tradicional, puesto que, por primera vez, todos los alumnos

1. Cecilia García Huidobro et. al, A estudiar se aprende, México, Alfaomega, 2005.

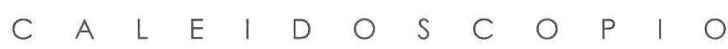


de propedéutico recibieron capacitación sobre la utilización de las bases de datos. Sin embargo, se requiere que los profesores soliciten a sus alumnos trabajos o tareas de fuentes o referencias científicas. En consecuencia, la capacitación para el uso de la biblioteca virtual o tradicional debe extenderse a los profesores. Asimismo deberán fomentar el hábito de la lectura.

Continuar y formalizar el seguimiento sobre demanda de los estudiantes con mayor necesidad de mejorar sus aptitudes de comunicación oral y escrita, programa educativo que surgió a partir de la problemática detectada en el cuatrimestre propedéutico, a través de las materias de Métodos de estudio y de Comunicación oral y escrita.

Complementar o ampliar el enfoque y alcance de las tutorías que actualmente tienen una dirección centrada en la dimensión emocional, de formación en valores humanos y de planeación de vida, hacia uno que implique la asimilación y uso de métodos eficaces de estudio, de acuerdo a los estilos propios de aprendizaje.

Las distintas academias deberán asumir el compromiso de promover el uso de herramientas de aprendizaje significativo a fin de lograr integrar la teoría y la práctica, los conocimientos, habilidades, actitudes, por tanto aprender a aprender, aprender a ser y aprender a hacer. Lo anterior se puede visualizar en las planeaciones de clase y operar con las distintas actividades de aprendizaje.

Actualizar la metodología para el desarrollo del proceso de en señanza aprendizaje efectivo, es decir, se requiere realizar el diseño instruccional con el propósito de captar el interés de los alumnos como un medio para lograr los resultados de aprendizaje esperados. Asimismo, es necesario llevar a cabo una evaluación integral que implique la identificación del nivel de dominio en cada disciplina, retroalimentar acerca del logro o las áreas de oportunidad a trabajar y así orientar a los alumnos de manera clara y concreta sobre lo que deben reforzar o mejorar.

Por Academia, ampliar el acervo de material didáctico para facilitación del proceso de enseñanza y aprendizaje.

La Universidad Politécnica de Aguascalientes apuesta por una formación significativa integral, y sus planes y programas de estudio pasaron por un proceso de diseño basado en la educación basada en competencias. 
Este diseño debería verse reflejado en una percepción de parte de los estudiantes de un método diferente y sobre todo mejor, de parte de sus profesores universitarios, sin embargo la encuesta parece demostrar que lo que ellos perciben es justo una práctica tradicional, semejante al servicio que han recibido en sus escuelas previas.

Cuando se les preguntó qué fue lo que se evaluó en sus trabajos, destacan la presentación (siempre, 68\%), la coherencia y orden (siempre, 67\%) y la extensión (51\%), en contraste con ideas originales, capacidad de crítica, capacidad de análisis, capacidad de síntesis e incluso uso de bibliografía, categorías que difícilmente rebasan el $20 \%$. Esto nos habla del enfoque centrado en hacer producir grandes volúmenes al estudiante, sin un claro objetivo de qué capacidades se pretende estimular y desarrollar.

Al abordar la cuestión sobre lo que caracteriza al desempeño docente, desde la perspectiva del estudiante, de nuevo obtienen registros altos aspectos tradicionales como la asistencia a clases, claridad en la exposición, y los aspectos que contribuyen al aprendizaje significativo, como trabajo colectivo, relacionar los contenidos con problemas del entorno del estudiante o aceptar discusiones sobre los puntos de vista del propio docente de nuevo reciben puntuaciones bajas.

Un aspecto trascendente lo constituye la percepción de que la calificación final del curso se obtiene a través de un examen escrito (siempre, más del 70\%), mientras que las exposiciones grupales, autoevaluación, prácticas en campo prácticamente no son tomadas en cuenta para definir la evaluación final. Es claro que el método de evaluación incide en los métodos de estudio, ya que aquel exige el ejercicio de ciertas habilidades intelectuales y es por demás sabido que los exámenes escritos suelen estar centrados en elementos de memorización, más que en aptitudes intelectuales de nivel superior. Finalmente, los estudiantes declaran que en sus estudios se fomenta la creatividad y la cooperación entre estudiantes, aunque esto es poco congruente con los elementos antes mencionados, es posible que las actividades cotidianas del aula estimulen estos aspectos, pero no exista un sistema de trabajo docente que aproveche este factor.

Es claro que, pese al buen enfoque que tienen los planes y programas de la institución, la falta de capacitación para los docentes, el predominio poblacional de profesores de tiempo parcial sobre 
los de tiempo completo, y la propia preferencia de los estudiantes por métodos tradicionales de enseñanza, hacen que el aprendizaje significativo se dé más por trabajo aislado y hasta circunstancial de algunos docentes y estudiantes, más que por un trabajo intencionado y diseñado para ello.

Se requiere instrumentar y ejecutar un programa de capacitación a profesores sobre educación basada en competencias, rediseñar el proceso de evaluación de la función docente, con referencia al enfoque recién mencionado y diseñar e implantar un proceso de evaluación del aprendizaje que esté orientado al reconocimiento y estímulo de capacidades, habilidades, competencias, para provocar un cambio en los hábitos de los estudiantes de la UPA y promover el aprendizaje para la vida.

(3) 